\title{
Kearns' syndrome, a new form of cardiomyopathy
}

\author{
S. C. Uppal ${ }^{1}$ \\ From the Department of Cardiology, University Hospital, Utrecht, The Netherlands
}

\begin{abstract}
A 19-year-old boy was admitted with Adams-Stokes syndrome. Subsequent examination revealed bilateral exophthalmoplegia, ptosis, and retinal pigmentary change. A pacemaker was implanted because of complete heart block. Only I2 similar cases have been previously described.
\end{abstract}

The detection by Meerschwam of abnormalities in skeletal muscle in patients with hypertrophic obstructive cardiomyopathy has raised the question that this disease may represent a more generalized muscle disorder, but the matter is still unresolved (Goodwin, I970; Meerschwam, 1969).

Cardiomyopathy is a recognized entity in muscle dystrophies and myotonia dystrophica, where there is generalized degeneration of skeletal muscles (Welsh, Lynn, and Haase, 1963).

Sandifer described cardiomyopathy in association with external ophthalmoplegia (I946). Later, Kearns and Sayre (1958), and Kearns (1965) described 9 cases with external ophthalmoplegia, retinal pigmentary change, and complete heart block. The purpose of this communication is briefly to review the salient features of this interesting syndrome described by Kearns. Furthermore, haemodynamic data not hitherto reported, in a patient thought to have this syndrome, are presented. Kearns' syndrome deserves attention in cardiology because of the presence of complete heart block.

\section{Case report}

In 1963 a I2-year-old boy was referred to the Eye Hospital of the University of Utrecht because of embarrassing ptosis, which was surgically corrected. Six years later he was referred to a neurologist because of two 'black-outs' on the road. An electroencephalogram showed no evidence of epilepsy or other abnormalities. In March 1970, when 19 years old, he was admitted to the Cardiology Unit of the University Hospital in Utrecht, with a history of transient loss of consciousness while walking. Physical examination revealed a normally-developed boy with divergent strabismus, generalized limitation of all eyeball movements, and conspicuous ptosis; dark adaptation was normal. Fundus 1 Present address: Department of Cardiology, University
Hospital, Leiden, The Netherlands. oculi showed peripapillary pigment deposit giving a halo appearance but no evidence of retinitis pigmentosa.

In the cardiovascular system the heart rate was $44-72$ / $\mathrm{min}$, and the blood pressure $130 / 60 \mathrm{mmHg}$. The first sound was normal and followed by a grade $\mathrm{I} / 4$ protosystolic murmur without a definite point of maximal intensity. There was wide splitting of the second heart sound, both components being of equal intensity. At the apex, a third heart sound was heard. The rest of the physical examination revealed no abnormalities. There was no evidence of myasthenia, myotonia, or muscular hypertrophy elsewhere.

Laboratory findings Blood group A rhesus positive. The blood picture and ESR were normal. Twenty-fourhour creatinine excretion in urine $\mathrm{I} \cdot 2 \mathrm{mg}$ (low). Blood urea $52 \mathrm{mg} / \mathrm{r} 000 \mathrm{ml}$, creatinine I I $\mathrm{mg} / \mathrm{r} 000 \mathrm{ml}$, serum electrolytes, protein-bound iodine, liver function tests, lipid spectrum, blood sugar, serum aspartate aminotransferase and alanine transferase, lactic dehydrogenase, and creatine phosphokinase enzymes were all normal. Anti-heart muscle antibody was negative, antiskeletal muscle antibody doubtfully positive. Toxoplasma dye test positive in $1 / 512$, and complementfixation test positive in $\mathbf{I} / 8$. Rectal biopsy showed no evidence of amyloidosis.

Electrocardiogram (Fig. I) This showed sinus rhythm at 60 a minute: PR interval $0.20 \mathrm{sec}, \mathrm{QRS}$ duration $0.12 \mathrm{sec}$. There were periods of first-degree and second-degree heart block as well as periods of sinus arrest. The QRS complexes showed right bundle-branch block configuration with left axis deviation, compatible with partial bilateral bundle-branch block. The possibility of biventricular hypertrophy could not be excluded The chest $x$-ray revealed slight left ventricular enlargement.

Jugular venous tracing $Y$-descent deeper than $\mathrm{x}$ descent.

Carotid pulse tracing Normal. On cardiac catheterization - all intracardiac and intravascular pressures 


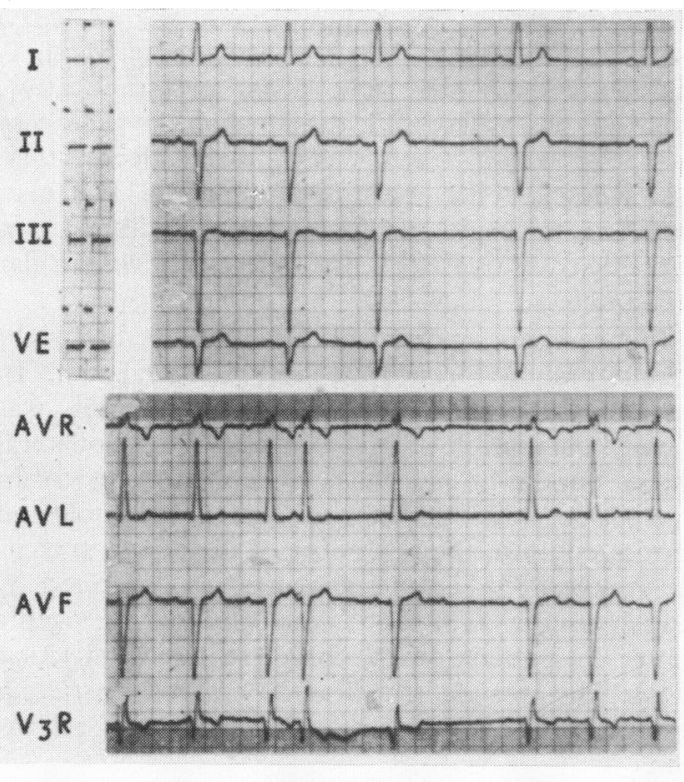

A

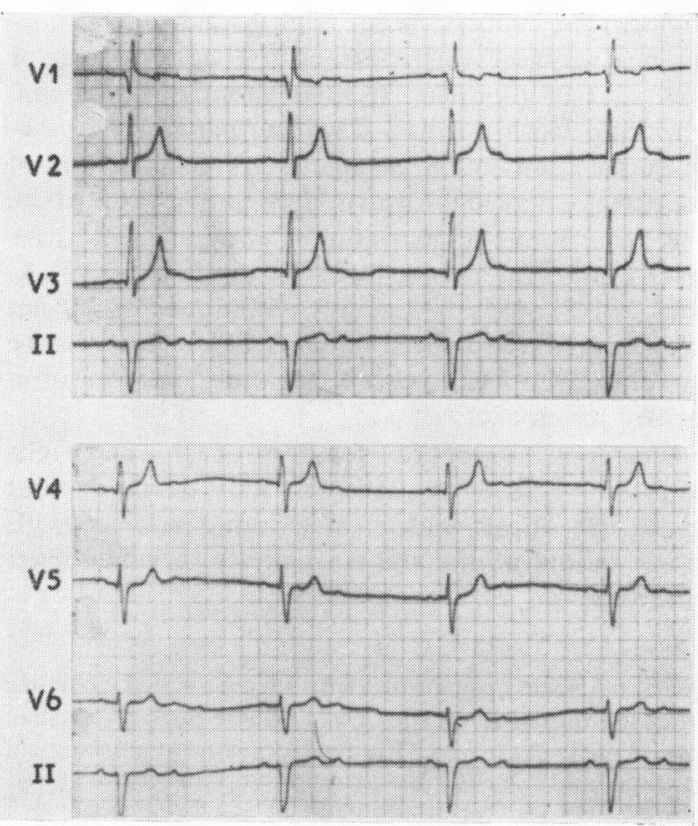

B

FIG. I Electrocardiogram. Record $A$ shows firstdegree heart block and period of sinus arrest. There is an extreme left axis deviation. Record $B$ shows a complete right bundle-branch block and a seconddegree heart block. were normal. The oxygen saturations were normal. The left ventricular end-diastolic pressure was $8 \mathrm{mmHg}$ and peak $\mathrm{dp} / \mathrm{dt}$ value $980 \mathrm{mmHg} / \mathrm{sec}$. The cardiac index was $3.61 . / \mathrm{m}^{2}$. There was no evidence of Ebstein's disease or intracardiac shunt.

Cineangiocardiography Injection of dye into the left ventricle showed slight mitral reflux during ventricular diastole. There were no premature beats during dye injection and no signs of hypertrophic obstructive cardiomyopathy or evident left ventricular wall thickening. On contrast injection into the root of the aorta no coronary arterial obstruction could be seen.

On audiometry a bilateral perceptive dip was demonstrable and there was no labyrinthine response to chlorethyl.

Cerebrospinal fluid Protein $124 \mathrm{mg} / \mathrm{roO} \mathrm{ml}$, on electrophoresis gammaglobulin is per cent, leucocytes 96, later 3. Erythrocytes nil.

Electroencephalogram Irregular with diffuse changes (abnormal).

Electromyogram (external ocular rectus muscle) Myopathic pattern.

Ocular fluorescein angiogram Besides tapetoretinal pigmentary changes there was also a tapetochoroidal component present especially in the peripapillary area. These findings do not fit into the classical tapetochoroidal dystrophies (Fig. 2).

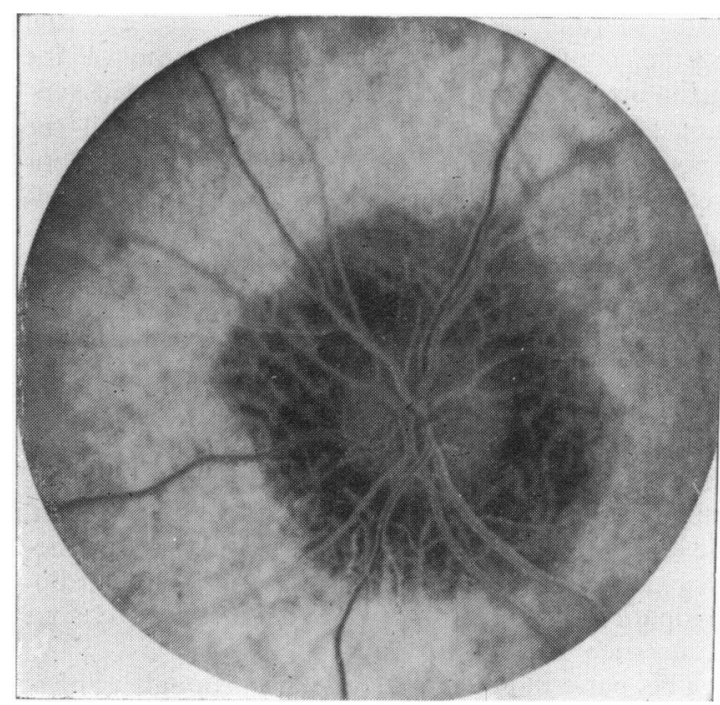

FIG. 2 Fluorescein ocular angiogram: showing peripapillary tapetochoroidal pigmentary change. 


\section{Progress}

During cardiac catheterization the patient developed Adams-Stokes attacks due to ventricular asystole and was temporarily paced with a transvenous bipolar catheter. The heart block did not respond to atropine or isoprenaline therapy. A pacemaker (Cordis) was later implanted. His ophthalmoplegia and ptosis have remained stationary since 1963 . He is doing well up to the time of publication of this paper.

\section{Discussion}

Summarizing, this patient has partial bilateral bundle-branch block with Adams-Stokes syndrome, an abnormal jugular venous tracing, slight diastolic mitral reflux, bilateral external ophthalmoplegia, bilateral ptosis, peripapillary retinal pigmentary change, asymptomatic labyrinthine disturbance, audiometric perceptive dip, raised gammaglobulin in the cerebrospinal fluid, an abnormal electroencephalogram, a myopathic-pattern electromyogram, and atypical tapetochoroidal dystrophy on fluorescein angiography. Chronic progressive external ophthalmoplegia was for the first time called ocular myopathy in a classic paper by Kiloh and Nevin (I95I), stressing the myopathic nature of the disease. In their series there was no cardiomyopathy. The first authentic association of external ophthalmoplegia and cardiomyopathy was described by Sandifer (1946). Marinesco (1910), in his monograph on diseases of muscle, asserts that of the muscles which offer extraordinary resistance to involvement by muscular dystrophy, the heart is the most notable. Kearns and Sayre (1958) published a series of nine cases under the heading: 'External ophthalmoplegia, pigmentary degeneration of the retina and cardiomyopathy: a new recognized syndrome'. Five out of these nine cases had all the foresaid three components; frequent associations were peripheral and facial muscle weakness, deafness, small stature, abnormal electroencephalogram, and increased protein content of the cerebrospinal fluid. The age of onset varied from 3 to 25 years, sex incidence being equal. He concluded that external ophthalmoplegia, cardiomyopathy, and peripheral muscle weakness were all a result of a generalized muscle disorder. Abnormal electroencephalogram and increased protein in the cerebrospinal fluid suggest central nervous involvement. He asserted that the association between ophthalmoplegia, retinal pigmentary degeneration, and cardiomyopathy does not appear to be merely a chance occurrence but a syndrome.

The patient described here has enough clinical features to justify a diagnosis of Kearns' syndrome. It is interesting to note that the cardiac output and end-diastolic pressure in the left ventricle are nor- mal. Furthermore, in the jugular venous tracing the $\mathrm{y}$-descent is deeper than the $\mathrm{x}$-descent, which is corroborated in the right atrial pressure curve. Normally the $x$-descent is deeper than the $y$-descent in jugular venous tracing. A y-descent deeper than the $\mathrm{x}$-descent has been described by Hartman (I960) in tricuspid insufficiency, atrial fibrillation, operated cases of atrial septal defect, and ventricular septal defect.

In five cases reported by Kearns, four had right bundle-branch block with left axis deviation. In the present case also a similar combination was found, compatible with a diagnosis of bilateral partial bundle-branch block (Rosenbaum, 1970). In this patient mitral reflux started in mid-diastole and continued up to the beginning of the next ventricular systole. This is best explained as described by Rutishauser et al. (1966) who reported atriogenic reflux in patients with complete atrioventricular block. This was explained on the basis of ventricular to atrial pressure gradient created by atrial relaxation.

This case is apparently the first of this syndrome in which the peripapillary retinal pigmentary change was analysed with the help of fluorescein angiography. There was striking choroidal fluorescence present which formed the background for fine piles of pigment. Retinal vascular filling and emptying were normal. This peripapillary tapetochoroidal pigmentary change is atypical for any of the tapetochoroidal dystrophies so far described.

Kearns forecast an ominous prognosis in these cases due to the problems of complete heart block. Now with modern pacing techniques one can offer new hope to these patients. The present case is doing well two years after implantation of a cardiac pacemaker.

Electrocardiographic features such as right bundle-branch block with left axis deviation and first-degree heart block in a young subject with ptosis should arouse the suspicion of a generalized syndrome.

I wish to express my gratitude to Professor F. L. Meijler for correction of the script, Dr. J. Wisse Smit for cardiac data, Dr. J. A. F. M. Luyten for expert neurological opinion, and Dr. C. H. Scheffer and Professor J. A. Oosterhuis for ophthalmological investigation and opinion.

\section{References}

Goodwin, J. F. (1970). Congesive and hypertrophic cardiomyopathies. A decade of study. Lancet, I, 73 I.

Hartman, H. (1960). The jugular venous tracing. American Heart fournal, 59, 698. 
Kearns, T. P. (1965). External ophthalmoplegia, pigmentary degeneration of the retina, and cardiomyopathy: a newly recognized syndrome. Transactions of the American Ophthalmological Society, 63, 559.

Kearns, T. P., and Sayre, G. P. (1958). Retinitis pigmentosa, external ophthalmoplegia, and complete heart block: unusual syndrome with histologic study in one of two cases. Archives of Ophthalmology, 60, 280.

Kiloh, L. G., and Nevin, S. (I95I). Progressive dystrophy of the external ocular muscles (ocular myopathy). Brain, 74, II5.

Marinesco, G. (1910). Maladies des muscles. Nouveau Traité de Médecine et de Thérapeutique, Fasc. 38, pp. 34 and I Io. J. B. Bailliere, Paris.

Meerschwam, I. S. (1969). Hypertrophic Obstructive Cardiomyopathy, p. I29. Excerpta Medica, Amsterdam.
Rosenbaum, M. B. (1970). The hemiblocks: diagnostic criteria and clinical significance. Modern Concepts of Cardiovascular Disease, 39, I4I.

Rutishauser, W., Wirz, P., Grander, M., and Luthy, E. (1966). Atriogenic diastolic reflux in patients with atrioventricular block. Circulation, 34, 807.

Sandifer, P. H. (1946). Chronic progressive ophthalmoplegia of myopathic origin. Fournal of Neurology, Neurosurgery and Psychiatry, n.s. 9, $8 \mathrm{I}$.

Welsh, J. D., Lynn, T. N., Jr., and Haase, G. R. (1963). Cardiac findings in 73 patients with muscular dystrophy. Archives of Internal Medicine, 112, 199.

Requests for reprints to Dr. S. C. Uppal, Department of Cardiology, University Hospital, Leiden, The Netherlands. 